compared between patients seen in the 12 months before the pandemic $(22 / 03 / 2019$ - 22/03/2020) with those seen in the 12 -months after $(23 / 03 / 2020$ - 23/03/2021), using non-parametric tests.

Results Overall, 190 patients (median age 43 years, $n=157$ (82.6\%) female, $41.0 \%$ IBS-C, $24.7 \%$ IBS-D, IBS-M $33.7 \%$ and $0.5 \%$ IBS-U) with refractory IBS were included. Compared to those seen in the 12-months before the pandemic $(n=107)$, the cohort seen during the Covid-19 pandemic $(\mathrm{n}=83)$ had a significantly higher median IBS-SSS (318 vs. 352, $p=0.03$ ), and more extra-intestinal symptoms (noncolonic score: 225 vs. $269, p=0.03)$. During the pandemic, median abdominal pain (50 vs. $63, p=0.05)$ and abdominal distension scores (60 vs. $75, p=0.008)$ were significantly higher, and patients that were unmarried had a higher median IBS symptom severity (IBS-SSS: 320 vs. $359, p=0.03$ ). Interestingly, those seen during the pandemic had more difficulty with sleep (53 vs. $68, p=0.03$ ), and more feelings of helplessness and loss of control (50 vs. 70, $p=0.02)$, compared to those seen before the pandemic. There was no difference in the levels of anxiety and depression between the pre and post lockdown groups (HAD-Anxiety: 11 vs. 11.5, $p=0.96$; HADDepression: 8 vs. $8, p=0.84)$.

Conclusions This study has shown for the first time that patients seen in tertiary care with refractory IBS during the covid-19 pandemic had a significantly higher symptom burden emphasising the importance of gut-brain axis in IBS. Furthermore, lack of support and perceived loss of control appear to be contributory factors.

\section{OWE-1 INVESTIGATION OF THE METABOLOME AND MYCOBIOME IN PARKINSON'S DISEASE}

${ }^{1}$ Gerum Gashaw Gebeyehu*, ${ }^{1}$ Luke Flain, ${ }^{1}$ Rachael Slater, ${ }^{1}$ Alessandra Frau, ${ }^{2}$ Chris Probert, ${ }^{3}$ Umer ljaz, ${ }^{2}$ Eduardo De Pablo-Fernandez, ${ }^{2}$ Tom Warner. ${ }^{1}$ University Of Liverpool, Liverpool, UK; ${ }^{2}$ UCL Queen Square Institute of Neurology, London, UK; ${ }^{3}$ University of Glasgow, Glasgow, UK

\subsection{6/gutjnl-2021-BSG.32}

Introduction The brain-gut axis is implicated in the pathogenesis of Parkinson's disease (PD). The 'hibernating spore' hypothesis proposes that the aetiological agent for PD is a fungal agent or its metabolite - possibly originating from a dysbiosis of the gut mycobiome. We evaluate the faecal metabolome and mycobiome in PD by assessing volatile organic compounds (VOCs) and highly-conserved fungal rRNA genes, respectively.

Methods VOCs were extracted from faecal samples from 35 PD patients and two groups of controls $(n=35$ and $n=15)$ using solid phase micro-extraction and gas chromatography and mass spectrometry. DNA was extracted from a subset of 24 PD and 20 control samples: 18S rRNA gene amplicons were prepared and sequenced using an Illumina MiSeq platform. Metabolomics and mycobiome statistical analysis were carried out using Metaboanalyst and $\mathrm{R}$ respectively. The $\mathrm{R}$ package mixOmics was used for integrated omics analysis in this subset.

Results After correction for multiple comparisons, 6 VOCs were associated with PD and 12 with the primary control group (FDR < 0.025). 1,3-ditert-butylbenzene, $\gamma$-Terpinene, $\alpha$ Pinene, O-Cymene and D-Limonene were more abundant in PD patients; short chain fatty acids were less abundant. The PD mycobiome had a higher alpha-diversity; patients with

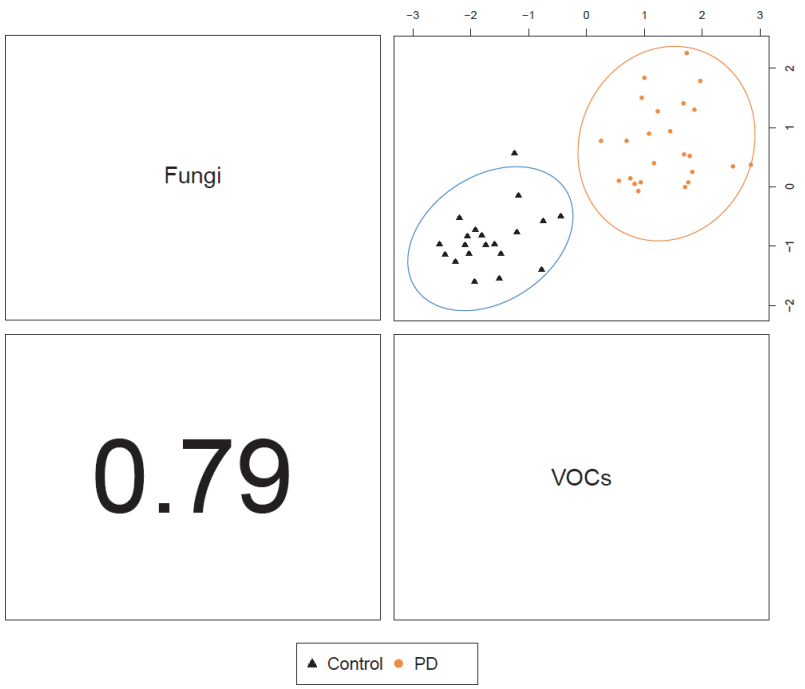

Abstarct OWE-1 Figure 1 Multi-omics sPLS-DA scatterplot comparing $\mathrm{PD}$ and controls. There were strong overall correlations between VOCs and fungi in PD and in controls (Pearson's correlation coefficient in lower left plot)

worse PD-related constipation had a significantly lower mycobiome richness $(\mathrm{P} \leq 0.05)$. Beta-diversity measures found a significant difference between the mycobiomes of PD and controls. Uncultured Tremellales, Hanseniaspora, Kazachstania and Penicillium genera were more abundant in PD whereas Saccharomyces genera were less abundant (FDR $<0.0007$ ). MixOmics analysis showed that VOCs and fungi were able to separate $\mathrm{PD}$ and control groups- there were correlations between VOCs and fungi in both PD and control groups ( $\mathrm{r}=$ 0.79) (Figure 1). MixOmics found Torulaspora delbrueckii was associated with PD and two VOCs - $\alpha$-Pinene and $\beta$-pinene. Conclusion PD patients had a distinct metabolome and mycobiome compared to healthy controls. There are interrelated changes of metabolome and mycobiome, suggesting a role for fungal dysbiosis and subsequent metabolome changes in the pathogenesis of PD.

\section{Gastroenterology service}

\section{OFR-1 YOUNG PERSONS AND HEALTH CARE PROFESSIONALS EXPERIENCE OF VIRTUAL GASTROENTEROLOGY CONSULTATIONS - A MULTICENTRE SURVEY}

${ }^{1}$ Rhona Hubbard*, ${ }^{2}$ Naila Arebi, ${ }^{3}$ Alenka Brooks, ${ }^{4}$ Sara El-Kouli, ${ }^{4}$ Fevroni Kiparissi, ${ }^{5}$ Ella Mozdiak, ${ }^{6}$ Philip Smith, ${ }^{7}$ Natalia Zarate-Lopez, ${ }^{8}$ Vikki Garrick, ${ }^{1}$ Priya Narula. ${ }^{1}$ Sheffield Children's Hospital NHS Foundation Trust, Sheffield, UK; ${ }^{2}$ Northwick Park St Mark's Hospital, London, UK; ${ }^{3}$ Sheffield Teaching Hospitals NHS Foundation Trust, Sheffield, United Kingtom; ${ }^{4}$ Great Ormond Street Hospital for Children NHS Foundation Trust, London, UK; ${ }^{5}$ University Hospitals of North Midlands NHS Trust, Stoke-on-Trent, UK; ${ }^{6}$ Royal Liverpool University Hospital, Liverpool, UK; ' University College London Hospitals, London, UK; ${ }^{8}$ Glasgow Children's Hospital, Glasgow, UK

\subsection{6/gutjnl-2021-BSG.33}

Introduction During COVID many clinic consultations moved to a virtual platform. Young people (YP) require additional considerations in this setting. The aim of this study was to explore the experiences of YP and Healthcare Professionals (HCP) to establish whether developmentally appropriate care can be delivered effectively via virtual clinics (VC). 